\section{Cannabis auf Rezept könnte 2017 kommen \\ Hüftschmerz mit Algorithmus klären}

Wenn Bundesgesundheitsminister Hermann Gröhe Recht behält, werden schwerkranke Patienten im Frühjahr 2017 die Möglichkeit haben, Cannabis vom Arzt auf Kassenrezept verordnet zu bekommen. Denn spätestens zu diesem Zeitpunkt wird das Gesetz zur Änderung betäubungsmittelrechtlicher und anderer Vorschriften in Kraft treten. Voraussetzungen für die Verordnung sind z.B. schwerwiegende Erkrankungen, keine Alternativen zur Behandlung mit Cannabisarzneimitteln, die Aussicht auf eine spürbare positive Beeinflussung auf den Krankheitsverlauf oder auf schwerwiegende Symptome und die Teilnahme der Patienten an einer anonymisierten Begleitforschung.
Beim Pädiatrie-Update-Seminar 2016 in Köln hat der Orthopäde PD Dr. Holger Mellerowicz einen praxistauglichen Algorithmus zur Abklärung von Hüftschmerzen im Kindes- und Jugendalter vorgestellt: Mit Humpeln oder Belastungsverweigerung einhergehende akut auftretende Hüftschmerzen sind die klinischen Leitsymptome der Coxitis fugax. Zur wichtigsten Differenzialdiagnose zählt z.B. die septische Arthritis. Mittels der vier Prädiktoren CRP, Leukozytenzahl, Gehfähigkeit und Körpertemperatur ist es nach Einschätzung von Mellerowicz mit hoher Wahrscheinlichkeit möglich, die Coxitis fugax von septischen Arthritiden abzugrenzen.

Pädiatrie-Update-Seminar, Köln 2016

\section{Vielfältiger Schmerz}

Das individuelle Schmerzempfinden ist so vielfältig wie die Ursachen, aus denen es resultiert. Am Beispiel des Rückenschmerzes wird dies deutlich: Nicht nur biomechanische, auch arbeitsplatzbezogene, psychosoziale und viele andere Faktoren können den Schmerz bedingen und aufrecht halten. Dabei kommt dem subjektiven Körperbild nach neuen Erkenntnissen eine wichtige Funktion im Krankheitsprozess zu. Erste Ansätze zu „Körperbildtherapien" scheinen demnach vielversprechend. Erfahren Sie mehr - im Schwerpunkt „Schmerz"!

Claudia Daniels Redakteurin

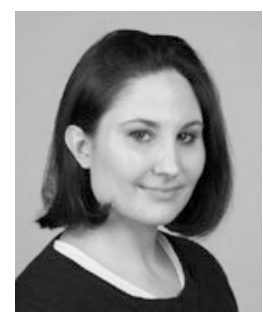

\title{
Mit Chili-Schärfe gegen starke Schmerzen
}

Capsaicin kann bekanntlich bei Schmerzen helfen, etwa der Post-Zoster-Neuralgie. Häufig kommt es jedoch zu Nebenwirkungen wie starkem Brennen. Forscher haben nun eine Substanz ausfindig gemacht, die sich ebenso eignen könnte, um starke Schmerzen zu lindern - jedoch weitaus verträglicher ist: Ein Team hat die Substanz Capsazepin, das den Capsaicin-Rezeptor teilweise blockiert, genauer untersucht. Der Stoff hatte in Studien anderer Wissenschaftler Colitis ulcerosa bei Mäusen verhindert. Jedoch musste dafür eine unbe- kannte Nebenwirkung von Capsazepin verantwortlich sein. Die Forscher erlebten eine Überraschung: Der Wirkstoff hemmte den Rezeptor nicht, sondern aktivierte ihn höchst effektiv. Dies führte dazu, dass er gegen den Reizstoff unempfindlich wurde. Die schützende Wirkung von Capsazepin bestand also darin, dass die Nozizeptoren auf entsprechende Reize weniger reagierten und keine entzündungsfördernden Neuropeptide mehr freisetzten.

Sci Rep 2016; doi:10.1038/srep28621

\section{Rektusdiastase verursacht keine Rückenbeschwerden}

Eine Rektusdiastase als physiologische Veränderung während der Schwangerschaft sollte sich in den Monaten nach der Entbindung von selbst wieder schließen. Doch offenbar hält die Diastase bei vielen Frauen noch lange nach der Geburt des Kindes an. Darauf weisen die Ergebnisse einer prospektiven Kohortenstudie hin, an der 300 Erstgebärende beteiligt waren. Gemessen wurde der Rektusabstand in der 21. Schwangerschaftswoche sowie sechs Wochen, sechs Monate und ein Jahr post partum. Die Diastaseprävalenz betrug dabei rund 33\%, 60\%, 45\% und 33\%. Frauen, die schwer heben mussten, waren häufiger betroffen. Negativ verlief dagegen die Suche nach einer oft vermuteten Assoziation von Rektusdiastase und Kreuzschmerzen. Beschwerden dieser Art hatten 46\% der Frauen mit und 33\% der Frauen ohne Diastase. Ein signifikanter Unterschied bestand nicht.

Sperstad JB et al. Br J Sports Med 2016; 50: 1092-1096

\section{Schmerzarm pieksen}

Impfungen sind notwendig, schon bei den Allerkleinsten. Nicht selten sind sie begleitet von Schmerz und Stress. Die Akzep$\operatorname{tanz}$ für weitere Impfungen sinkt. Dem hat die STIKO nun Rechnung getragen. In ihren aktuellen Empfehlungen 2016/2017 gibt sie konkrete Hinweise zur Schmerzund Stressreduktion: So sollte z.B. schon die Nadellänge sorgfältig gewählt werden. Werden mehrere Injektionen zum gleichen Zeitpunkt verabreicht, sollte die schmerzhafteste Injektion, etwa die Pneumokokken- oder MMR-Impfung, zuletzt injiziert werden. Auch mit Maßnahmen zur Schmerzlinderung lassen sich Impfungen erträglicher machen. Zwischen dem vierten Lebensmonat und dem zwölften Lebensjahr können in Einzelfällen schmerzstillende Medikamente eingesetzt werden, etwa Lidocain-haltige Schmerzpflaster oder -cremes. Möglich ist auch ein Eisspray, das rasch wirkt. Gerade bei Kindern kommt Ablenkungsmanövern ein hoher Stellenwert zu. Nuckeln an einem Schnuller oder Stillen kommt dabei ebenso in Betracht wie die Applikation einer Glukoselösung oder einer anderen süßen Flüssigkeit. Um Stressreaktionen zu mindern, ist es außerdem hilfreich, die Eltern frühzeitig aufzuklären.

112. Jahrestagung der DGKJ, Impfungen, 17.9.2016 